\title{
Expanding Flexibility with P2H for Integrated Energy Systems
}

Wang, Tinghua; Zhu, Xiaojun; Ge, Pudong; Hu, Qinran; Wu, Qiuwei; Dou, Xiaobo; Ding, Haohui

\section{Published in:}

Proceedings of IET RPG 2019

Link to article, DOI:

$10.1049 /$ cp.2019.0670

Publication date:

2020

Document Version

Peer reviewed version

Link back to DTU Orbit

Citation (APA):

Wang, T., Zhu, X., Ge, P., Hu, Q., Wu, Q., Dou, X., \& Ding, H. (2020). Expanding Flexibility with P2H for Integrated Energy Systems. In Proceedings of IET RPG 2019 Institution of Engineering and Technology. https://doi.org/10.1049/cp.2019.0670

\section{General rights}

Copyright and moral rights for the publications made accessible in the public portal are retained by the authors and/or other copyright owners and it is a condition of accessing publications that users recognise and abide by the legal requirements associated with these rights.

- Users may download and print one copy of any publication from the public portal for the purpose of private study or research.

- You may not further distribute the material or use it for any profit-making activity or commercial gain

- You may freely distribute the URL identifying the publication in the public portal 


\title{
EXPANDING FLEXIBILITY WITH P2H FOR INTEGRATED ENERGY SYSTEMS
}

\author{
Tinghua Wang ${ }^{1}$,Xiaojun $\mathrm{Zhu}^{1}$,Pudong $\mathrm{Ge}^{2}$, Qinran $\mathrm{Hu}^{2 *}$,Qiuwei $\mathrm{Wu}^{3}$,Xiaobo $\mathrm{Dou}^{2}$,Haohui Ding ${ }^{2}$ \\ ${ }^{1}$ Economic Research Institute, State Grid Jiangsu Electric Power Company, Nanjing, China \\ ${ }^{2 *}$ Southeast University, 2 Sipailou, Nanjing, China \\ ${ }^{3}$ Technical University of Denmark, 2800 Kgs. Lyngby, Denmark \\ *qhu@seu.edu.cn
}

Keywords: INTEGRATED ENERGY SYSTEMS, OPERATING FLEXIBILITY, WIND CURTAILMENT

\begin{abstract}
Combined heat and power (CHP) unit serves as a significant kind of heating source in energy systems, but its heat production must be accompanied by electric production. It could worsen the wind power curtailment in the context of large-scale installed wind farms, which is unfriendly to the integrated energy system operation. Power to hydrogen $(\mathrm{P} 2 \mathrm{H})$, a promising energy conversion technology, is a possible solution to mitigate this issue by consuming excessive electric power, and stored hydrogen can be used in many fields, such as fuel cell and chemical synthesis. Hence, this paper designs an integrated energy system optimisation model with $\mathrm{P} 2 \mathrm{H}$ to discuss the feasibility of combining electric power, heat and hydrogen together. Water electrolysis is modelled with an electricity-hydrogen conversion efficiency factor, which is easy to be integrated into a mixed integer optimization problem that mimics the system operation in a 24-hour period. Three cases are designed to demonstrate that the application of $\mathrm{P} 2 \mathrm{H}$ improves operating flexibility, and more specifically, the flexibility is visualized by showing equivalent operating region of CHP units.
\end{abstract}

\section{Introduction}

Until the year 2017, the total installed capacity of wind power in China reached nearly $200 \mathrm{GW}$, which shared $35 \%$ of that around the world [1]. The problem under this background is that wind power integration is often limited, and large wind energy generation is curtailed due to lacking enough realtime wind consuming capacity in today's energy system [2].

There are some literatures that investigated the possible solutions to wind integration. Since combined heat and power (CHP) plants, which is inflexible, are satisfying heating demands while generating electricity [3] and in winter CHPs normally operate in heat-demand-driven mode and produce electricity as well, which leads to poor wind power utilization rate [4][5], introducing effective ways to enhance the operational flexibility of CHPs can exert positive influences on wind power integration. Heat storage tanks (heat accumulators) could increase the flexibility [5] and the benefit of heat storage is sensitive to the structure of existing heat supply system and to the level of wind penetration [6]. Installing electric boilers [7] and heat pumps [8] to produce heat by consuming electricity can increase the feasible operational region of CHPs, and it is a possible solution to reduce wind power curtailment.

Power to hydrogen $(\mathrm{P} 2 \mathrm{H})$ is also a possible and meaningful solution to consume excessive electric power, as well as meet potential hydrogen demand from the traffic sectors. Hydrogen is produced through low-cost environmentally clean processes by means of renewable energy sources [9]. $\mathrm{P} 2 \mathrm{H}$ can consume excessive power production from wind generations [10][11]. Many scholars have done a large amount of research on $\mathrm{P} 2 \mathrm{H}$, and the research detailed the characteristics of $\mathrm{P} 2 \mathrm{H}$ [12], such as electrochemical submodel and thermal submodel.

Although these technologies expand the flexibility of energy systems, these works have not fully investigated the operating characteristics of combined power, heat and hydrogen systems. Therefore, this paper proposes an integrated power, heat and hydrogen optimization (IPHHO) model for multi-energy suppliers to explore the flexibility of integrated energy systems improved by electric boilers and P2H systems. By implementing the IPHHO model, this paper will analyse 1) operating performance of the integrated energy system coupled to electric boilers and $\mathrm{P} 2 \mathrm{H}$ systems; 2) improvement in flexibility of CHP units, which visualizes the enhanced flexibility of the whole system.

The rest of this paper is organised as follows: An integrated power, heat and hydrogen optimization (IPHHO) model is detailed in Section 2. Section 3 elaborates on the expansion of CHP operational region with multi-energy suppliers. Simulation results are analysed in Section 4. Finally, Section 5 concludes the presented work.

\section{IPHHO model formulation}

This section designs an integrated power, heat and hydrogen optimization (IPHHO) model to mimic the operation of integrated energy systems with the consideration of different constraints in system operation including load balance, ramping limits, etc. It optimizes the hourly power, heat and hydrogen output of all controllable generation resources with the objective to minimize the operation costs for the multienergy supplier. The configuration of the integrated energy system is shown in Fig. 1. 


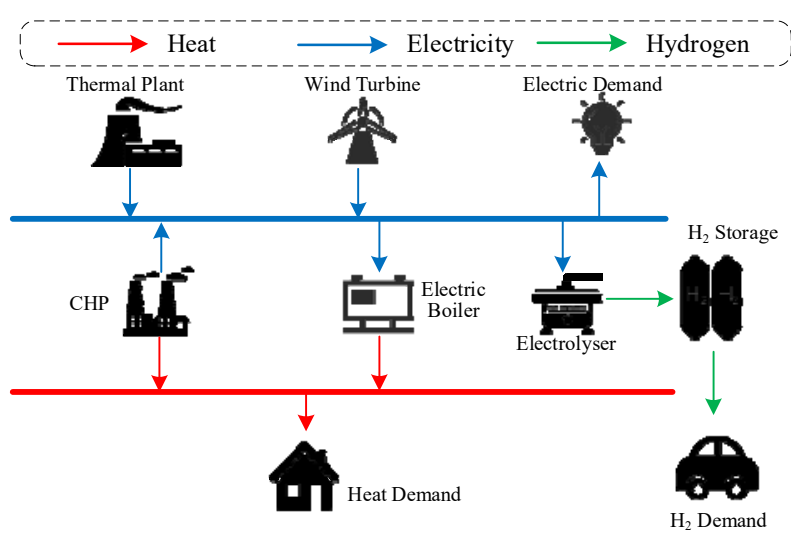

Fig. 1 Configuration of the integrated energy system

\subsection{Decision variables and objective function}

The decision variables include energy generation for power, heat and hydrogen generation for each unit at every time period: 1) $u_{i, t}$ represents $\mathrm{ON} / \mathrm{OFF}$ status of unit $i \in\{\mathcal{T P}, \mathcal{C H} \mathcal{P}\}$ at time $t$;2) $p_{i, t}$ represents electric power generation of unit $i \in\{\mathcal{T} \mathcal{P}, \mathcal{C H} \mathcal{P}, \mathcal{W}, \mathcal{E B}, \mathcal{E} \mathcal{L}\}$ at time $t$ (MW); 3) $q_{i, t}$ represents heat power generation of unit $i \in\{\mathcal{C H} \mathcal{P}, \mathcal{E B}, \mathcal{E} \mathcal{L}\}$ at time $\$ \mathrm{t} \$(\mathrm{MW}) ; 4) n_{i, t}$ represents Hydrogen power generation of unit $i \in\{\mathcal{E} \mathcal{L}, \mathcal{H} \mathcal{S}\}$ at time $t$ (kg). It is noteworthy that $i \in \mathcal{T P}$ represents thermal power plant unit $i$, and $\mathcal{T P}, \mathcal{C H} \mathcal{P}, \mathcal{W}, \mathcal{E B}, \mathcal{E} \mathcal{L}, \mathcal{H S}$ represent thermal power plant, combined heat and power, wind generation, electric boiler, electrolyser, hydrogen storage units respectively in the following sections. Other variables associated with the status of the CHP units are also included in the model.

The proposed model is designed to minimize the fuel consumption, as well as wind power curtailment. The objective function includes the total fuel cost of all thermal power plants and CHP units, as well as the penalties for curtailment of wind generation[2]:

$$
f=\min \sum_{t=1}^{T_{h}} \sum_{i \in \mathcal{T P}} p_{i, t} \cdot r_{i}+\sum_{t=1}^{T_{h}} \sum_{i \in \mathcal{C H} \mathcal{P}} c_{i, t}+\beta \sum_{t=1}^{T_{h}} \sum_{i \in \mathcal{W}}\left(\widehat{P_{i, t}}-p_{i, t}\right)
$$

where the cost of a conventional thermal power plant unit is modelled as linear to its energy output, the price rate of unit $i$ is $r_{i} . T_{h}$ represents total hourly time steps. The cost of CHP units will be introduced in the following section. $\beta$ and $\widehat{P_{i, t}}$ represent penalty factor for wind curtailment and predicted maximum electric power output of unit $i$ respectively.

\subsection{System constraints}

\subsubsection{P2H system modelling}

A typical $\mathrm{P} 2 \mathrm{H}$ unit is made up of the electrolyser and the hydrogen tank. The water electrolysis process has been discussed exactly in [12], and there are three kinds of electrolysers, namely alkaline water electrolyser, proton exchange membrane electrolyser and solid oxide electrolyte electrolyser. The accurate model of electrolysis is nonlinear and complicated, which makes the whole energy system difficult to simulate as a convex optimisation problem. For the sake of simplification, we employ the key energy conversion process with a conversion efficiency denoted as $\eta_{i}, i \in \mathcal{E} \mathcal{L}$.

According to [13], an electrolyser produces hydrogen when the electric power delivered by electric generation units is higher than demand. Applying Faraday's law, the hydrogen molar flow of the electrolyser can be expressed as:

$$
n_{i, t}=\frac{\eta_{i} p_{i, t} \Delta t}{\mathrm{LHV}_{\mathrm{H}_{2}}}, i \in \mathcal{E} \mathcal{L}
$$

where $\mathrm{LHV}_{\mathrm{H}_{2}}$ represents Lower heating value of $\mathrm{H}_{2}$.

As for the hydrogen tank, an important control variable is the hydrogen tank pressure, which can be expressed by the ideal gas law [13]:

$$
\Delta P_{i, t}=\frac{R T_{i}}{V_{i}} n_{i, t}, i \in \mathcal{H S}
$$

where $R$ denotes the universal gas constant, and $T_{i}, V_{i}$ denote working temperature and volume of unit $i \in \mathcal{H} \mathcal{S} . P_{i, t}$ denotes tank pressure of unit $i \in \mathcal{H S}$ at time $t$, and the pressure has its limits:

$$
\underline{P_{i}} \leq P_{i, t} \leq \overline{P_{i}}
$$

It is noteworthy that the ideal gas law can be used by setting a relatively low maximum hydrogen pressure (13.8 bar) and the average temperature inside the hydrogen tank $T_{i}$ is assumed to be constant (313 K) [13].

\subsubsection{CHP modelling}

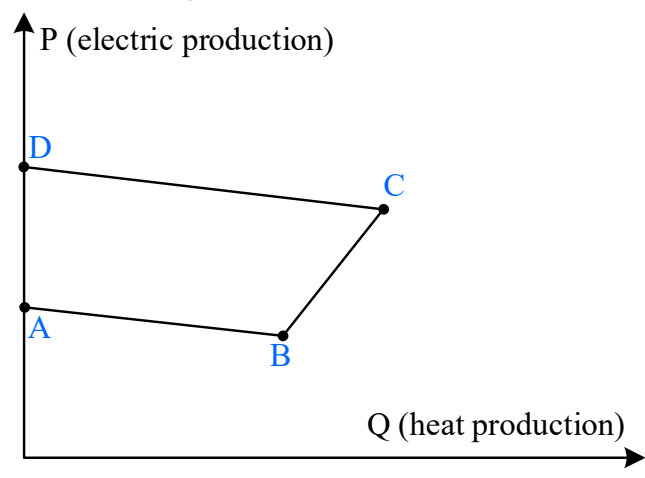

Fig. 2 Feasible operating region of a typical CHP

Feasible operating region, as shown in Fig. 2, has been widely adopted to describe the operation characteristics of a CHP unit. The heat and electric power production for CHP units are coupled, and electric power and heat outputs of the CHP units with convex feasible operational area as follows [5]: 


$$
\left\{\begin{array}{l}
q_{i, t}=\sum_{k=1}^{K_{i}} \alpha_{i, t}^{k} x_{i}^{k} \\
p_{i, t}=\sum_{k=1}^{K_{i}} \alpha_{i, t}^{k} y_{i}^{k} \quad, i \in \mathcal{C H P} \\
c_{i, t}=\sum_{k=1}^{K_{i}} \alpha_{i, t}^{k} z_{i}^{k} \\
\sum_{k=1}^{K_{i}} \alpha_{i, t}^{k}=1,0 \leq \alpha_{i, t}^{k} \leq 1
\end{array}\right.
$$

where $K_{i}$ denotes the number of boundaries of CHP unit $i$.

\subsubsection{Electric boiler modelling}

Heat produced by electric boiler $i$ has a linear relationship with the electric power consumed by this boiler.

$$
\begin{aligned}
& q_{i, t}+\eta_{i} p_{i, t}=0, i \in \mathcal{E B}, \forall t \\
& 0 \leq-p_{i, t} \leq \bar{P}_{i}, i \in \mathcal{E B}, \forall t
\end{aligned}
$$

where the conversion efficiency is denoted as $\eta_{i}, i \in \mathcal{E B}$.

\subsubsection{Electric power, heat and hydrogen balances}

The energy balance is formulated in equation (2). The system demand equals the sum of electric power, heat and hydrogen generated from all units subtracting the energy consumption. Please note $p_{i, t}, n_{i, t}$ are negative when unit $i$ consumes electric power and hydrogen respectively.

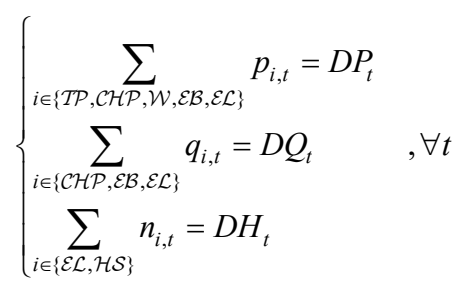

where $D P_{t}, D Q_{t}$ and $D H_{t}$ denote system demand for electric power, heat and hydrogen respectively at time $t$.

\subsubsection{Flexibility constraints}

There are ramping constraints for conventional thermal power plants and CHP units. Based on [2][4], for $\forall t, i \in \mathcal{T P} \cup \mathcal{C H} \mathcal{P}$, these constraints are as follow:

$$
\begin{aligned}
& p_{i, t}-p_{i, t-1} \leq R U_{i} \cdot u_{i, t-1}+S U_{i} \cdot\left(u_{i, t}-u_{i, t-1}\right) \\
& p_{i, t}-p_{i, t-1} \geq-R U_{i} \cdot u_{i, t-1}-S D_{i} \cdot\left(u_{i, t}-u_{i, t-1}\right) \\
& \underline{P_{i}} \cdot u_{i, t} \leq p_{i, t} \leq \overline{P_{i}} \cdot u_{i, t}
\end{aligned}
$$

Based on [2][4], the constraints of minimum ON and OFF time are formulated as follow:

$$
\sum_{t=1}^{G 1_{i}}\left(1-u_{i, t}\right)=0
$$

$$
\begin{gathered}
\sum_{t=j}^{j+U T_{i}-1} u_{i, t} \geq U T_{i} \cdot\left(u_{i, j}-u_{i, j-1}\right), \\
\forall j \in\left[G 1_{i}+1, T_{h}-U T_{i}+1\right] \\
\sum_{t=j}^{T_{h}}\left(u_{i, t}-\left(u_{i, j}-u_{i, j-1}\right)\right) \geq 0, \forall j \in\left[T_{h}-U T_{i}+2, T_{h}\right] \\
\sum_{t=1}^{G 0_{i}} u_{i, t}=0
\end{gathered}
$$

$$
\begin{aligned}
& \sum_{i=j}^{j+D T_{i}-1}\left(1-u_{i, t}\right) \geq D T_{i} \cdot\left(u_{i, j-1}-u_{i, j}\right), \\
& \forall j \in\left[G 0_{i}+1, T_{h}-D T_{i}+1\right] \\
& \sum_{t=j}^{T_{h}}\left(1-u_{i, t}-\left(u_{i, j-1}-u_{i, j}\right)\right) \geq 0, \\
& \forall j \in\left[T_{h}-D T_{i}+2, T_{h}\right]
\end{aligned}
$$

Equations (9)-(11) describes the ON time limit, where $U T_{i}$ is the minimum ON time of unit $i, G 1_{i}$ is associated with the initial status of unit $i$ presenting the consecutive ON time intervals required before it can be shut down. Meanwhile, Equations (12)-(14) describes the OFF time limit, where $D T_{i}$ is the minimum OFF time of unit $i, G 0_{i}$ is associated with the initial status of unit $i$ presenting the consecutive OFF time intervals required before it can be start up.

\section{Operating analysis of integrated energy systems}

To better visualize the enhanced flexibility, the extra flexibilities from electrical boilers and electrolysers are shown as equivalent operating region expansion for CHP units. The equivalent electric power and heat production are expressed as:

$$
\begin{aligned}
& p_{i, t}^{\prime}=\sum_{i \in\{\mathcal{C H P}, \mathcal{E B}, \mathcal{E L}\}} p_{i, t} \\
& q_{i, t}^{\prime}=\sum_{i \in\{\mathcal{C H P}, \mathcal{E B}, \mathcal{E L}\}} q_{i, t}
\end{aligned}
$$

The expanded boundaries for the equivalent heat and electric power production of a CHP unit coupled to an electrolyser and an electrical boiler are shown in Fig. 3.

The electric boiler integrated into the system expands the maximum heat production, and the minimum electric power production decreases because electricity from $\mathrm{CHP}$ can be used to produce heat, as shown in Fig. 3 (a). Due to the similar electricity consumption, the expanded boundary caused by electrolyser has similarities to that of electric boiler. However, the electrolyser provide hydrogen instead of heat, so only electric production decreases and heat 
production does not change. With both equipment, the overall expanded operating region is shown in Fig. 3 (c).

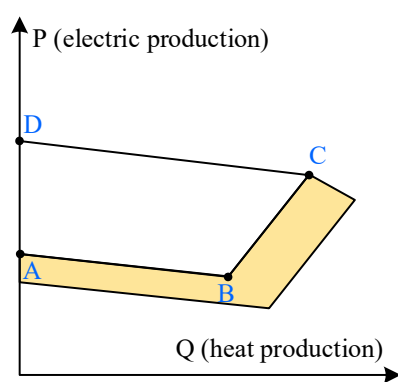

(a)

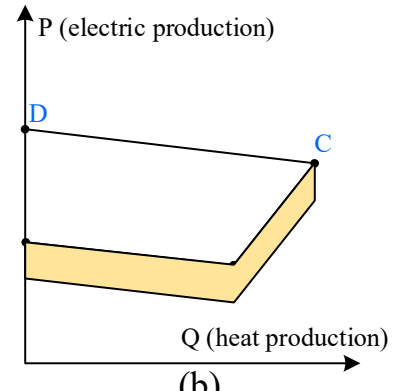

(b)

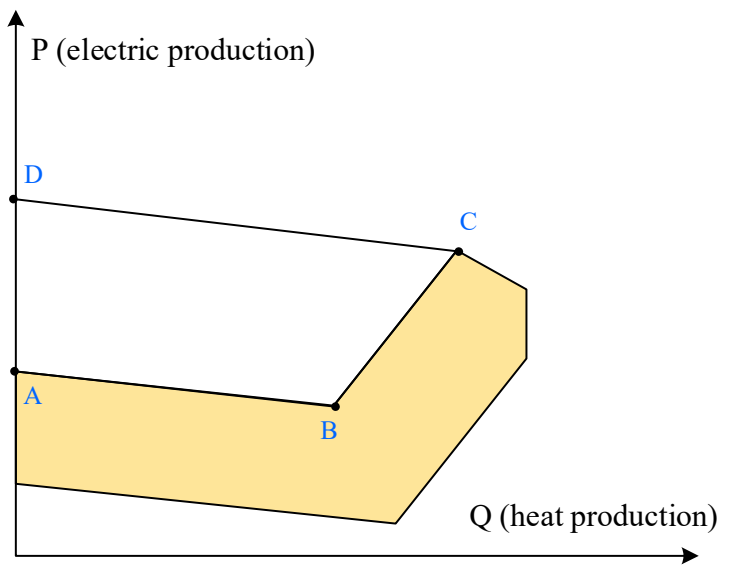

(c)

Fig. 3 Expanded operating region of a typical CHP

\section{Results}

In this section, a typical energy system is designed to evaluate the proposed IPHHO model for the integrated energy system including $\mathrm{P} 2 \mathrm{H}$.

\subsection{Test system introduction}

A test system is used to demonstrate the influence and effect of electrolyser and hydrogen storage coupled to the integrated energy system. In the system, one CHP along with a 20-MW electric boiler serves heating district. In addition, a 20-MW electrolyser also serves the system.

The hourly electric demand, wind generation, heat demand and hydrogen demand of the test system are shown in Fig. 4. The electric demand, wind generation and hydrogen demand are selected to show the representative cases and have the similar daily trend with [14]. The hydrogen demand data is derived from [15].

\subsection{Simulation results}

\subsubsection{System operating performance analysis}

The cost of operation is 352.9341, which is also the fuel cost. The optimal schedule is as shown in Fig. 5. In this case, the penalty for wind curtailment is 0 . In other words, all the electric power generated from wind generations are consumed in real time. Hydrogen tank, as a device for energy storage, stores energy when the electric power is higher than demand through electrolysis, and electric boiler, as an alternative heat source consumes provide extra heat by consuming electric power.
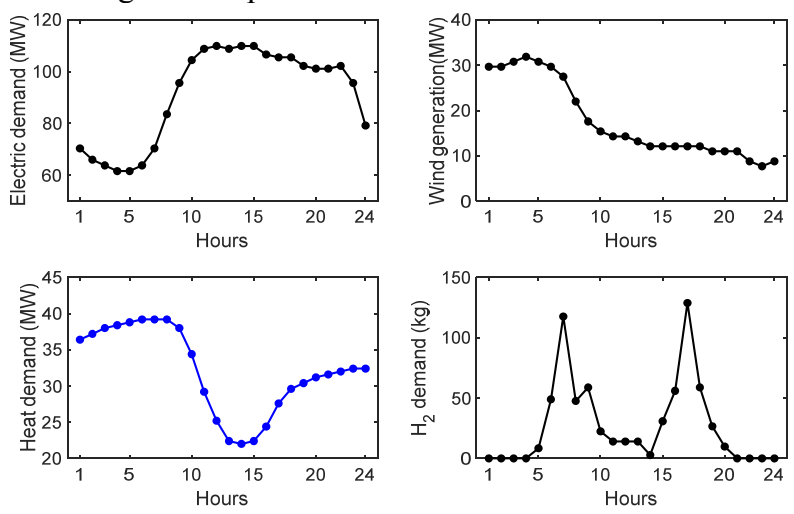

Fig. 4 System demand and wind power output
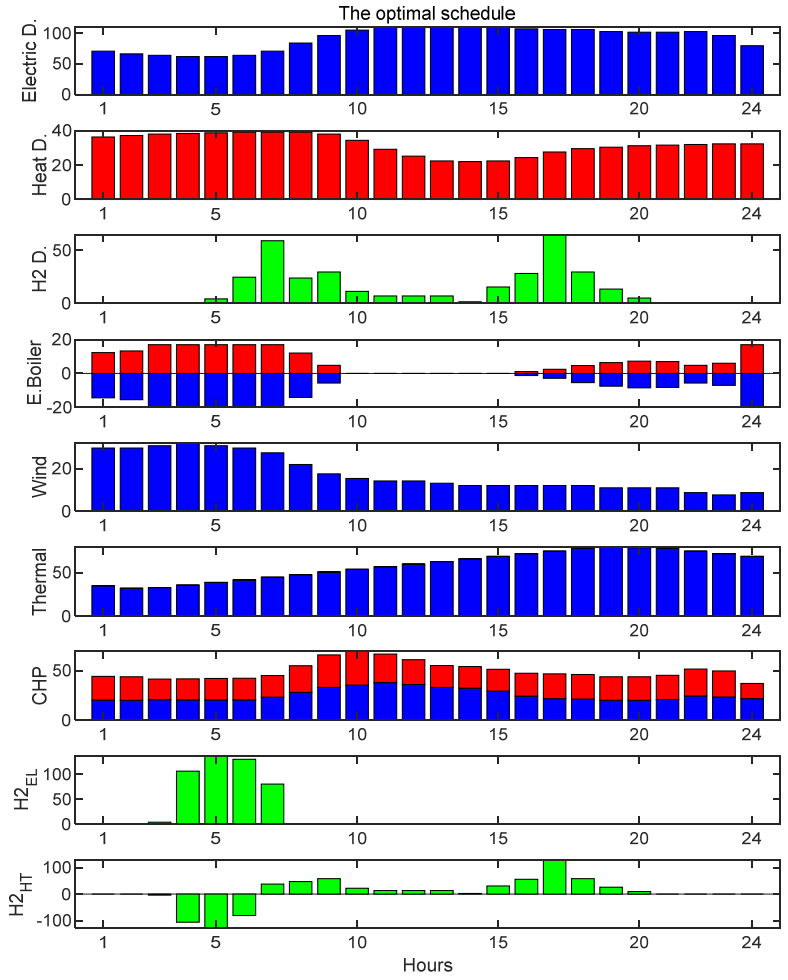

Fig. 5 The optimal schedule of the integrated system

\subsubsection{Improvement in flexibility of CHPs}

To illustrate the effectiveness in increasing the system flexibility by introducing $\mathrm{P} 2 \mathrm{H}$ and electric boiler, we design 3 cases to compare the system operating performance. In case 1 , the electric boiler and the hydrogen system do not serve the system. Only the electric boiler operating in the system in case 2 while both serve the system in case 3 .

The hourly electric power generated by different suppliers for each case is shown in Fig. 6, and the corresponding 
equivalent operating area of CHP is shown in Fig. 7. When only CHP serves as a heating producer, the heat output of CHP1 is restricted to about 40 MW from 1 am to 9 am due to no other alternative heating sources, so the electric output cannot be low in this off-peak time period, as shown in Fig. 7 (a). In addition to low electric load and high wind generation, large wind curtailment is inevitable.

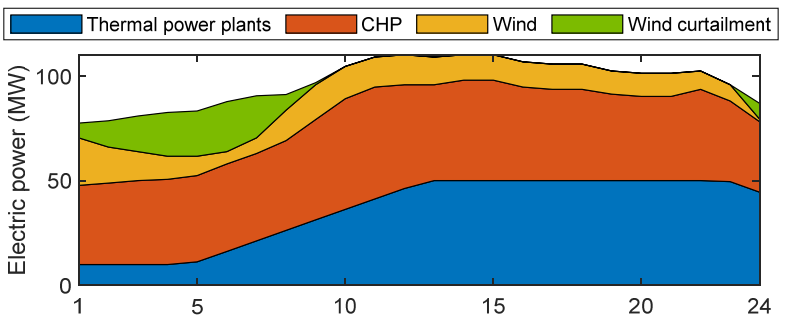

(a)

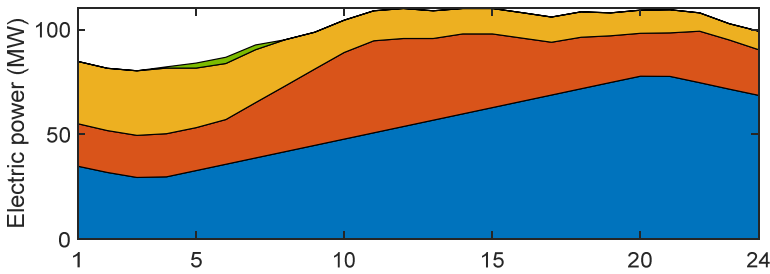

(b)

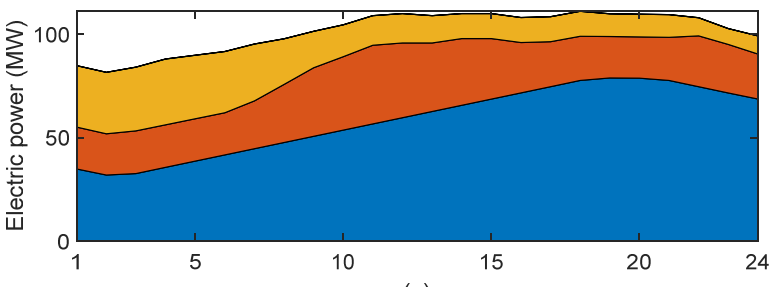

(c)

Fig. 6 Electric power balances: (a)case 1, (b) case 2, (c) case 3

When electric boiler is coupled to the system, its capability of consuming electricity to produce heat decreases 20 -MW heat output from CHP and gives an opportunity for wind integration in the off-peak hours, so the curtailed wind power goes down a large amount, as shown in Fig. 6 (b). In this case, the equivalent boundary of CHP is extended and the power output is reduced to about $5 \mathrm{MW}$ at off-peak hours, as shown in Fig. 7 (b)

The electrolyser in the $\mathrm{P} 2 \mathrm{H}$ system also makes it possible to increase the wind power integration into the system, although it cannot generate heat power. Hence, comparing to electric boiler, the $\mathrm{P} 2 \mathrm{H}$ system creates less system flexibility margin, as shown in Fig. 7 (c).

\section{Conclusion}

This paper proposes an integrated power, heat and hydrogen optimisation (IPHHO) model, where the operational cost of multi-energy suppliers and wind power utilization rate are considered, to explore the flexibility of integrated energy systems improved by electric boilers and $\mathrm{P} 2 \mathrm{H}$ systems. First,
IPHHO is implemented to mimic the system daily operation. Then, the improvement in flexibilities of CHP units, which represents the flexibility of the integrated system, is analysed. Finally, several cases are designed to illustrate the effectiveness of the proposed model.

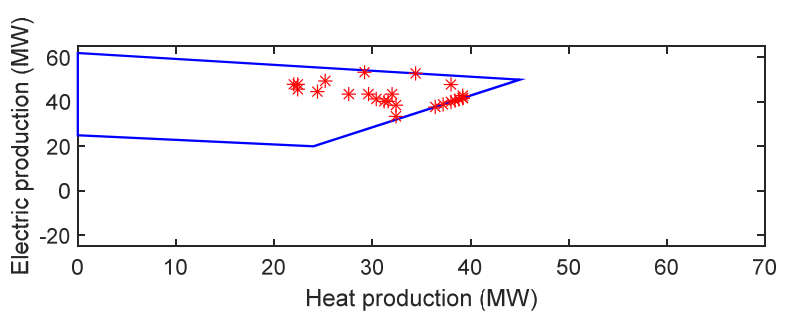

(a)

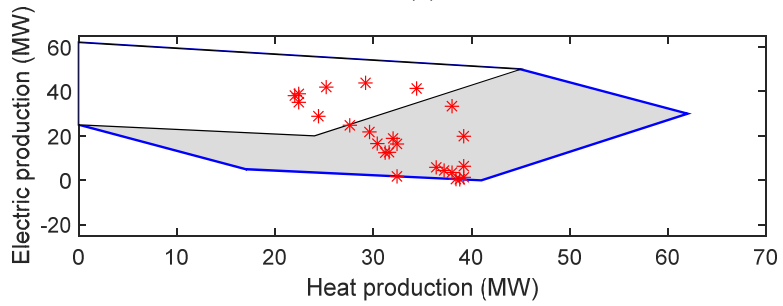

(b)

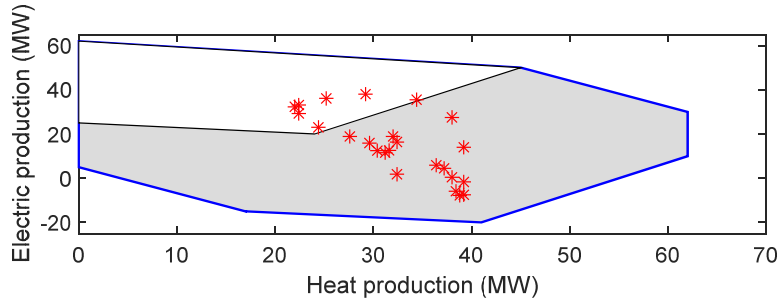

(c)

Fig. 7 Equivalent operating points and boundaries of CHP: (a) case $1,(\mathrm{~b})$ case 2 , (c) case 3

\section{Acknowledgements}

This research is supported by supported by the research and development program of State Grid Jiangsu Electric Power Company(J2019054), State Key Laboratory of Smart Grid Protection and Control, and the Fundamental Research Funds for the Central Universities (2242019K40017).

\section{References}

[1] 'Global wind report 2017', https://gwec.net/, accessed 25 April 2018

[2] Chen, X., McElroy, M.B., Kang, C.: 'Integrated energy systems for higher wind penetration in china: Formulation, implementation, and impacts', IEEE Transac- tions on Power Systems, 2018, 33, (2), pp. 1309-1319

[3] Benam, M.R., Madani, S.S., Alavi, S.M., et al.: 'Optimal configuration of the chp system using stochastic programming', IEEE Transactions on Power Delivery, 2015, 30, (3), pp. 1048-1056

[4] Li, Z., Wu, W., Shahidehpour, M., et al.: 'Combined heat and power dispatch con- sidering pipeline energy 
storage of district heating network', IEEE Transactions on Sustainable Energy, 2015, 7, (1), pp. 12-22

[5] Chen, X., Kang, C., O'Malley, M., et al.: 'Increasing the flexibility of combined heat and power for wind power integration in china: Modeling and implications', IEEE Transactions on Power Systems, 2015, 30, (4), pp. 1848-1857

[6] Hedegaard, K., Mathiesen, B.V., Lund, H., et al.: 'Wind power integration using individual heat pumpsanalysis of different heat storage options', Energy, 2012, 47, (1), pp. 284-293

[7] Meibom, P., Kiviluoma, J., Barth, R., et al.: 'Value of electric heat boilers and heat pumps for wind power integration', Wind Energy: An International Journal for Progress and Applications in Wind Power Conversion Technology, 2007, 10, (4), pp. 321-337

[8] Papaefthymiou, G., Hasche, B., Nabe, C.: 'Potential of heat pumps for demand side management and wind power integration in the german electricity market', IEEE Transactions on Sustainable Energy, 2012, 3, (4), pp. 636-642

[9] Ursua, A. , L. M. Gandia , and P. Sanchis.: 'Hydrogen Production From Water Electrolysis: Current Status and Future Trends', Proceedings of the IEEE, 2012, 100, (2), pp. 410-426

[10] Fu, C., Lin, J., Song, Y., et al.: 'Model predictive control of an integrated energy microgrid combining power to heat and hydrogen'. 2017 IEEE Conference on Energy Internet and Energy System Integration (EI2), Beijing, 2017, pp. 1-6.

[11] Li, J., Lin, J., Song, Y., et al.: 'Operation optimization of power to hydrogen and heat (p2hh) in adn coordinated with the district heating network', IEEE Transactions on Sustainable Energy, 2018, pp. 1-12

[12] Koponen, J.: 'Review of water electrolysis technologies and design of renewable hydrogen production systems'. Msc thesis, Lappeenranta University of Technology, 2015

[13] Cau, G., Cocco, D., Petrollese, M., et al.: 'Energy management strategy based on short-term generation scheduling for a renewable microgrid using a hydrogen storage system', Energy Conversion and Management, 2014, 87, (107), pp. 820-831

[14] '6 bus Data DR', http://motor.ece.iit.edu/data/6bus_Data_DR.pdf

[15] El-Taweel, N.A., Khani, H., Farag, H.E.Z.: 'Hydrogen storage optimal schedul- ing for fuel supply and capacity-based demand response program under dynamic hydrogen pricing, IEEE Transactions on Smart Grid, 2018, pp. 1-12 